\title{
Germline Nuclear-predominant Pten Murine Model Exhibits Impaired Social and Perseverative Behavior, Microglial Activation, and Increased Oxytocinergic Activity
}

Nick Sarn

Cleveland Clinic

Stetson Thacker

Cleveland Clinic https://orcid.org/0000-0001-6050-409X

Hyunpil Lee

Cleveland Clinic

Charis Eng ( $\nabla$ engc@ccf.org )

Case Western Reserve University School of Medicine https://orcid.org/0000-0002-3693-5145

\section{Research}

Keywords: PTEN mutation, autism spectrum disorder, mouse model, social impairment, microglia, oxytocin

Posted Date: October 30th, 2020

DOI: https://doi.org/10.21203/rs.3.rs-97746/v1

License: (c) (i) This work is licensed under a Creative Commons Attribution 4.0 International License. Read Full License 


\section{Abstract}

\section{Background}

Autism spectrum disorder (ASD) has a strong genetic etiology. Germline mutation in the tumor suppressor gene PTEN is one of the best described monogenic risk cases for ASD. Animal modeling of cell-specific Pten loss or mutation has provided insight into how disruptions to the function of PTEN affect neurodevelopment, neurobiology, and social behavior. As such, there is a growing need to understand more about how various aspects of PTEN activity, cell-compartment-specific functions, contribute to certain neurological or behavior phenotypes.

\section{Methods}

To understand more about the relationship between Pten localization and downstream effects on neurophenotypes, we generated the nuclear-predominant $P \operatorname{ten}{ }^{Y 68 H /+}$ mouse. We subjected the $P \operatorname{ten}{ }^{Y 68 H /+}$ mouse to morphological and behavioral phenotyping, including the three-chamber sociability and marble burying tests. We subsequently performed in vivo and in vitro cellular phenotyping and concluded the work with a transcriptomic survey of the $\mathrm{Pten}^{\mathrm{Y} 68 \mathrm{H} /+}$ cortex, which profiled gene expression.

\section{Results}

Despite no significant changes in downstream canonical Pten signaling, we found that the $P \operatorname{ten}^{Y 68 H /+}$ mouse presents with macrocephaly, social impairment (i.e., decreased sociability, decreased preference for novel social stimuli, and increased perseverative activity), with significant microglial activation accompanied by enhanced phagocytosis. Because of lack of canonical signaling alterations, we turned to analyzing the neural transcriptomes, which revealed overexpression of many genes involved in neuroinflammation and neuronal function, including oxytocin. Oxytocin transcript was 5-fold overexpressed $(P=0.0018)$ and oxytocin protein was strongly overexpressed in the $P t e n^{Y 68 H /+}$ hypothalamus.

\section{Conclusions}

The nuclear-predominant $P \operatorname{ten}^{Y 68 \mathrm{H} /+}$ model has clarified that Pten dysfunction links to microglial pathology and that timed decreased in Pten levels is the provoking insult. Notably, we demonstrate that Pten dysfunction associates with changes in the oxytocin system, an important connection between a prominent ASD risk gene and a potent neuroendocrine regulator of social behavior. Ultimately, the findings from this work may reveal important biomarkers and/or novel therapeutic modalities that could be explored in individuals with germline mutations in PTEN with ASD.

\section{Background}

It is well established that germline mutations in PTEN predispose individuals to autism spectrum disorder (ASD) and ranks among the most common monogenic etiologies [1-13]. We and others have shown that $7-27 \%$ of individuals with ASD and concurrent macrocephaly harbor germline mutations in PTEN, which 
when extrapolated across all cases of ASD may account for $0.5-5 \%$ of those cases $[5,7,13-16]$. In addition to ASD, germline PTEN mutations cause subsets of Cowden syndrome (CS, OMIM \#158350), BannayanRiley Ruvalcaba Syndrome (BRRS), Proteus syndrome (OMIM \#176920), and Proteus-like syndrome [17]. Irrespective of clinical syndrome and pathophysiology, anyone diagnosed with a germline PTEN mutation carries the molecular diagnosis of PTEN Hamartoma Tumor Syndrome (PHTS, OMIM \#601728) $[17,18]$.

PTEN has been well characterized as a tumor suppressor gene that removes the 3 ' phosphate group from phosphatidylinositol(3,4,5)-triphosphate (PIP3), thereby inhibiting the PI3K/AKT/mTOR signaling pathway, a major growth, survival, and migration pathway $[17,19,20]$. Beyond this canonical PTEN function, there is a growing body of research exploring the protein phosphatase and non-catalytic activities of PTEN [21,22]. The subcellular localization of PTEN and its importance to neurological phenotypes is of special interest. Recent work suggests that missense mutations in PTEN that disrupt subcellular localization may be more correlated with neurological phenotypes, specifically those associated with ASD-like behaviors [23-25]. Moreover, it has been observed that missense mutation versus other types of mutation are enriched among individuals with ASD [26-29]. However, there are still outstanding questions about the exact impact of PTEN localization on neurological structure and function.

In order to interrogate the effects of PTEN mislocalization, we developed two complementary mouse models of germline Pten mutation. One model exhibits cytoplasmic-predominant expression of Pten, the $P_{t e n}{ }^{m 3 m}$ model, while the other exhibits nuclear-predominant expression of Pten, the $\operatorname{Pten}^{Y 68 H}$ model. In the cytoplasmic-predominant model, we found no significant morphological or behavioral changes in Pten $^{\text {m3m4/t }}$ mice, but in the homozygous $\operatorname{Pten}^{m 3 m 4 / m 3 m 4}$ mice we observed dramatic macrocephaly and a sex-dependent increase in social motivation with severe deficits in motor coordination [23]. Additionally, we performed extensive cellular phenotyping on the $\operatorname{Pten}^{m 3 m 4}$ model, finding hypertrophy of neuronal somas, astrogliosis, dysmyelination, stunted maturation of neural stem cells (NSCs), precocious differentiation of oligodendrocyte progenitor cells (OPCs), and microgliosis, specifically cell-autonomous microglial activation and increased phagocytic response [23,26,30-32]. Our molecular characterization of the $P t e n^{m 3 m 4}$ model included an RNA sequencing experiment that found that the neural transcriptome included many genes relevant to human idiopathic ASD [24]. In contrast to the cytoplasmic-predominant model, the nuclear-predominant Pten mutant has never been characterized or subject to study. The $\mathrm{Y} 68 \mathrm{H}$ mutation, which induces nuclear-predominant Pten expression, especially in metabolically stressed cells [33,34], is likely to affect neurological function as it is a mutation that has been identified in PHTS patients diagnosed with ASD. Therefore, we hypothesize that the nuclear-predominant Y68H mutation in Pten contributes to deficiencies in social behavior as well as pathological changes in neuronal and glial function, but perhaps via a different mechanism.

\section{Methods}

\section{Animals}

We generated $\mathrm{Pten}^{\mathrm{Y} 68 \mathrm{H} /+}$ mice on a C57BL/6J (Jackson Laboratory, Bar Harbor, Milwaukee) background by introducing one missense mutation into exon three of the mouse Pten gene, specifically Pten c.202 T>C, via 
standard cre-lox methodology (Fig. 1A). This mutation targets the sequence analogous to the ATP-binding motif B found in human PTEN[33,34]. Mice were backcrossed onto C57BL/6J (Jackson Laboratory) inbred strain for five generations to reduce the impact of genetic heterogeneity on the results. Genotyping was performed on genomic DNA from clipped toes per the Jackson Laboratory protocol using modified PCR primers: Y68H F1, 5'-GTTTCACAGCTGGTTGGAAGG -3', and Y68H R1, 5'-TGTACCCAGCTCACAGACTTCC -3'. Mice were maintained on a 14:10 light: dark cycle with access to food and water ad libitum. The room temperature was maintained between 18 and $26^{\circ} \mathrm{C}$. Animals were euthanized via $\mathrm{CO}_{2}$ asphyxiation followed by cervical dislocation. All experiments were conducted under protocols approved by the Institutional Animal Care and Use Committee (IACUC) at Cleveland Clinic. Additionally, for all experiments described, we utilized only male mice for our experiments

\section{Western blot analysis}

Cortical regions of the brain were isolated, snap-frozen, and stored at $-80^{\circ} \mathrm{C}$. Tissue was thawed on ice and lysed in RIPA buffer (10 mM Tris-Cl [pH 8], 1 mM EDTA, 0.5 mM EGTA, 1\% Triton X-100, 0.1\% sodium deoxycholate, $0.1 \%$ SDS, $140 \mathrm{mM} \mathrm{NaCl}$ ), containing phosphatase inhibitor \#2 (Sigma, St. Louis, Missouri, \#P5726-5ML), phosphatase inhibitor \#3 (Sigma, \#P0044-5ML), and protease inhibitor (Sigma, \#P8345$5 \mathrm{ML}$ ). Lysates were quantified for protein concentration using bicinchoninic acid assay (BCA) assay, equalized to a concentration of $1 \mu \mathrm{g} / \mu \mathrm{l}$ of protein per sample, and finally $20 \mu \mathrm{g}$ of protein was loaded to a 4-15\% gradient polyacrylamide gel for SDS-PAGE separation. The separated proteins were transferred to a nitrocellulose membrane and blocked overnight at $4^{\circ} \mathrm{C}$ in $3 \%$ bovine serum albumin (BSA) in $1 \mathrm{X}$ Trisbuffered saline, containing $0.2 \%$ Tween-20 (TBST). Membranes were then washed with TBST and incubated with experiment-specific primary antibodies diluted in bovine serum albumin (BSA) overnight at $4^{\circ} \mathrm{C}$. The following antibodies were used: PTEN (1:5000, \#ABM-2025, Cascade Bioscience, Winchester, Massachusetts), IBA1 (1:500, \#019-19741, Wako, Bellwood, Virginia), MBP (1:1000, MAB386, EMD Millipore, Burlington, Massachusetts), PLP (1:1000, ab28486, Abcam, Cambridge, Massachusetts), GAPDH (1:5000, 2118L, Cell Signaling, Danvers, Massachusetts), HSP90 (1:1000, 4874, Cell Signaling), Lamin A/C (1:1000, 2032, Cell Signaling), Beta-actin (1:5000, AM4302, Thermo-Fisher, Waltham, Massachusetts), phospho-AKT Ser473 (1:1000, 9271, Cell Signaling), AKT (1:1000, 4691, Cell Signaling), phospho-ERK1/2 (1:1000, 9101, Cell Signaling), ERK1/2 (1:1000, 9102, Cell Signaling), phosph-S6 (1:1000, 4858S, Cell Signaling), and C1q (1:500, ab71940, Abcam). We removed the primary antibody solution and performed three washes, 10 minutes per wash, with TBST. Blots were probed with goat anti-mouse secondary antibody IRDye800CW (1:20,000, \#213965, LI-COR, Lincoln, Nebraska) or goat anti-rabbit IRDye680 (1:20,000, \#213971, LI-COR) diluted in BSA, for two hours at room temperature. The membranes were washed three times, 10 minutes each in TBST, and imaged using the Odyssey CLx imaging system (LI-COR). Using ImageJ (National Institute of Health, Bethesda, Maryland, 1995), we performed densitometry analysis on these images to quantify protein expression.

\section{Behavior Testing}

To assess changes in social behavior, we employed the three-chamber sociability test according to a previously reported protocol $[23,35]$. Mice were placed in a center chamber for five minutes and then 
returned to their original cage. Next, the assessment consisted of a 10-minute trial, where the test mouse was returned to the central chamber and given a choice between two identical containers, one chamber containing a mouse, and the other an empty chamber. In order to measure preference for social novelty, the 10-minute trial was repeated two days later with a familiar mouse in one chamber and a novel mouse in the other. Time spent in each chamber and time spent in close contact with the containers were recorded and quantified using Noldus EthoVision software (Wageningen, Netherlands).

To assess repetitive behavior, we administered the marble burying test to our mice per a previously published protocol [36]. This trial is performed by placing 20 marbles atop clean bedding material and placing the trial mouse into the case for a 30-minute session. Upon completion of the trial the number of buried and non-buried marbles was scored.

\section{Primary microglia cell culture}

Mixed glia were obtained by trypsinization of postnatal day 2 (P2) cortices followed by plating on poly-Dlysine coated T-75 culture flasks. Mixed glia cultures, were maintained in DMEM (Lerner Research Institute Media Core, Cleveland, OH) with 10\% FBS and 1\% Penicillin and Streptomycin (Pen/Strep). Once the mixed glia cultures reached confluency at approximately DIV 10, they were agitated for one hour at $170 \mathrm{RPM}$. At this point, the supernatant was removed and spun down at 1200 RMP in order to isolate primary microglia. Isolated microglia were resuspended in DMEM with 10\% FBS and 1\% Pen/Strep and seeded on poly-Dlysine coated glass cover slips subsequently used for immunofluorescence staining and phagocytic assays at DIV 3 post-shaking.

\section{Phagocytosis assay}

We plated primary microglia at a density of $1 \times 10^{5}$ in a 12-well dish with PDL-coated coverslips for 48 hours in a $37^{\circ} \mathrm{C}$ cell incubator with $5 \% \mathrm{CO}_{2}$ and $100 \%$ humidity. Next, we blocked $1 \mu \mathrm{m}$ fluorescent beads (Sigma-Aldrich, \#L1030) in FBS for one hour at $37^{\circ} \mathrm{C}$ at a ratio of $1: 5 \mathrm{v} / \mathrm{v}$. Florescent beads were diluted with DMEM to reach a final concentration of $0.01 \%(\mathrm{v} / \mathrm{v})$. Microglial culture media was replaced with $250 \mu \mathrm{l}$ DMEM containing beads, and incubated for one hour at $37^{\circ} \mathrm{C}$ in a cell incubator. Cultures were washed thoroughly five times with ice-cold PBS (Lerner Research Institute Media Core) and fixed in ice-cold methanol prior to immunofluorescent staining for Iba1 (1:500, \#019-19741, Wako).

\section{Immunofluorescence staining of brain tissue}

Mice were euthanized and perfused with approximately $50 \mathrm{ml}$ of 1X PBS. Brain tissue was then extracted and fixed in $4 \%$ PFA $(\mathrm{pH}=7)$ for 24 hours at $4^{\circ} \mathrm{C}$. Brains were then washed three times with PBS and cryoprotected in $30 \%$ sucrose dissolved in PBS for 94 hours at $4^{\circ} \mathrm{C}$. Frozen brain sections were cut coronally to a width $10 \mu \mathrm{m}$ on a cryostat and mounted on polarized glass slides (Fisherbrand Superfrost Plus microscope slides, \#12-550-15, Fisher Scientific, Waltham, MA). OCT was removed by washing slides in PBS for 10 minutes and tissue was permeabilized with 3\% Triton-X dissolved in PBS for 10 minutes. Slides were next washed three times for five minutes each in PBS and probed with experiment specific primary antibodies: Iba1 (1:500, \#019-19741, Wako), Plp (1:1000, ab28486, Abcam), NeuN (1:250, MAB377, EMD 
Millipore), Olig2 (1:250, ab9610, Abcam), S100b (1:200, ab52642, Abcam), Gfap (1:250, sc-33673, Santa Cruz), Oxt (1:250, ab212193, Abcam), Pten (1:5000, \#ABM-2025, Cascade Bioscience) and incubated overnight at $4^{\circ} \mathrm{C}$. The following day, slides were washed with PBS for three times five minutes each. This was followed by incubation with secondary antibody for two hours: goat anti-mouse Alexa Fluor 568 (1:2000, \#A11031, Thermo-Fisher) and goat anti-rabbit Alexa Fluor 488 (1:2000, \#A11008, Thermo-Fisher). Post incubation, slides were washed and mounted in Vectashield medium with DAPI (Vector Laboratories, Burlingame, CA), coverslipped, and sealed with nail polish.

\section{In vitro immunofluorescence staining}

We cultured primary microglia on poly-D-lysine (PDL)-coated cover slips until DIV 14. Microglia were washed with ice-cold PBS and fixed in ice-cold methanol for two minutes. This was followed by three washes for five minutes each with ice-cold PBS. We then permeabilized the microglia with $0.03 \%$ Triton X100 dissolved in PBS for four minutes. Next, cells were blocked with $10 \%$ normal goat serum for one hour at room temperature, followed by incubation with primary antibody Iba1 (1:500, \#019-19741, Wako) diluted in $10 \%$ normal goat serum in PBS. Cells were then incubated in primary antibody overnight at $4^{\circ} \mathrm{C}$. The following day cells were washed with PBS three times for five minutes and secondary was added, goat antimouse Alexa Fluor 568 secondary antibody (1:2000, \#A11031, Thermo-Fisher) diluted in 10\% normal goat serum in PBS. The cells were incubated in secondary antibody for two hours at room temperature, washed with PBS three times for five minutes, and coverslipped with Vectashield medium with DAPI (Vector Laboratories).

\section{Immunofluorescence quantification}

We captured images of brain sections and primary microglia as confocal images using a Leica TCS-SP8AOBS inverted confocal microscope (Leica Microsystems, GmbH, Wetzlar, Germany). Brain sections and microglia cultures were imaged with a minimum of $\mathrm{N}=3$ biological replicates. Image J software was used to measure area and intensity of the stain and calculated integrated density of brain images. Additionally, ImageJ was used to measure area of stain per microglia in vivo to assess morphological changes.

\section{Transcriptomic data analysis}

We isolated total RNA from the cortex of eight $\mathrm{Pten}^{\mathrm{Y} 68 \mathrm{H} /+}$ mice and seven $\mathrm{Pten}^{+/+}$mice. Aliquots of roughly $60 \mathrm{ng} / \mu \mathrm{L}$ total RNA (average RIN score $=9.1$; Additional file 1: Table S1) were prepared (TruSeq Stranded Total RNA - RiboZero Gold, Illumina, San Diego, CA) and then sequenced using an Illumina NOVA-Seq. The resulting Fastq sequences were subject to standard processing and quality control (QC) evaluation, using MultiQC v1.9 (https://multiqc.info/). Then, we performed an alignment to a the mouse reference genome (mm10) using Spliced Transcripts Alignment to a Reference (STAR) 2.7.5 (https://github.com/alexdobin/STAR) [37-39] and repeated a quality control evaluation using MultiQC v1.9. One $\mathrm{Pten}^{+/+}$sample and three $\mathrm{Pten}^{\mathrm{Y68H/+}}$ samples were discarded due to a high proportion of repetitive sequences and generally poor alignment statistics (Additional file 1: Fig S1). Additionally, we used Salmon 1.8.0 (https://bioconductor.org/packages/release/workflows/html/rnaseqDTU.html) as an alternative method to count reads mapping to a present index of known cDNA transcripts. Subsequently, we 
performed DeSeq2 1.28.1 on STAR-aligned counts and Salmon-produced counts to assess differential expression (DE). These two methods were used to ensure concordance between both approaches. Genes experiencing DE were analyzed in RStudio 1.2.5001 using R 4.0.0 to construct volcano plots and heatmaps. Generally, a p-value $(P<0.05)$, fold change ( $\log _{2}($ Fold Change $) \geq 1.0$ or $\left(\log _{2}(\right.$ Fold Change $\left.) \leq-1.0\right)$, and count (RPKM > 10) thresholds were used for these analyses. In order to assess the biological impact of the DE results, we used STRING (https://string-db.org/) and Ingenuity Pathway Analysis (Qiagen, Redwood City, California) software.

\section{Statistical analysis}

We analyzed normally distributed data using a one-way analysis of variance (ANOVA) or Student's t-test, where appropriate (GraphPad Prism 8). After performing a one-way ANOVA (F), we performed a post-hoc Tukey-Kramer analysis. When data were not normally distributed, we performed non-parametric analyses including Mann-Whitney $\mathrm{U}$ and Kruskall-Wallis tests $(\mathrm{H})$, where appropriate (Graph Pad Prism 8). P-values that are less than 0.05 were considered statistically significant.

\section{Results}

\section{Pten $^{Y 68 H /+}$ mice exhibit increased nuclear Pten localization and increased brain mass}

We originally observed the PTEN ${ }^{Y 68 H}$ mutation in PHTS individuals diagnosed with ASD and found that this particular mutation was sufficient to disrupt the subcellular partitioning of PTEN protein, resulting in relatively predominant nuclear localization [34]. Furthermore, we generated the $\mathrm{Pten}^{\mathrm{Y} 68 \mathrm{H} /{ }^{+}}$mouse model by introducing a single missense mutation into exon three of mouse Pten (i.e., Pten c.202 T>C), thus converting tyrosine residue 68 into histidine (Fig. 1a). To assess the subcellular localization of Pten in our $\mathrm{Pten}^{\mathrm{Y} 68 \mathrm{H} / \mathrm{+}}$ mouse, we performed nuclear-cytoplasmic fractionation of cortical tissue from six-month-old mice and assessed protein localization via Western blot (Fig. 1b). We observed a decrease by visual inspection in Pten in the cytoplasmic fraction of $\mathrm{Pten}^{\mathrm{Y} 68 \mathrm{H} /+}$ hemibrain relative to $\mathrm{Pten}^{+/+}(\mathrm{N}=4$ mouse/genotype, Fig. 1b). Additionally, quantitative assessment showed that the ratio of nuclear-tocytoplasmic Pten is increased in the Pten ${ }^{Y 68 H /+}$ mice compared to $\operatorname{Pten}^{+/+}\left(\right.$Median $_{\Delta \mathrm{N} / \mathrm{CRatio}}=0.20 ; 97 \% \mathrm{Cl}$ : $0.15-0.34 ; P=0.029$; Fig. 1c). Next, we performed immunofluorescence staining for Pten in the brains of six-month-old $\mathrm{Pten}^{\mathrm{Y} 68 \mathrm{H} /+}$ mice, which also indicated an increase in the nuclear-to-cytoplasmic ratio of Pten expression by visual inspection (Fig. 1d). These observations are consistent with our Western data (Fig. 1b), both of which show enrichment of nuclear Pten expression relative to cytoplasmic Pten expression in the Pten $^{Y 68 H /+}$ brain (Fig. 1d).

Macrocephaly is a hallmark of PHTS individuals and all ASD in this setting is accompanied by macrocephly $[29,40,41]$; therefore to determine if a similar overgrowth phenotype exists in $\mathrm{Pten}^{\mathrm{Y} 68 \mathrm{H} / \mathrm{+}}$ mice, we performed a gross examination of $\mathrm{Pten}^{\mathrm{Y} 68 \mathrm{H} /{ }^{+}}$brains at six-months-of-age. We found a significant increase in brain mass as measured in grams in $\mathrm{Pten}^{\mathrm{Y} 68 \mathrm{H} /+}$ mice compared to $\mathrm{Pten}^{+/+}$littermate controls 
Median $\left._{\triangle \text { BrainMass }}=0.15 ; 97 \% \mathrm{Cl}: 0.090-0.21 ; P<0.0001\right)$ with no change in overall body weight (Fig. 1f) . These differences in brain mass are also apparent by visual inspection and displayed in Fig. 1e.

\section{$P t e n^{Y 68 H /+}$ mutant mice exhibit decreased sociability and repetitive behavior}

Next, we sought to examine the possibility that nuclear-predominant Pten expression in the central nervous system (CNS) may alter social behavior. Several studies have demonstrated that Pten loss, whether constitutional or conditional to the CNS, can have deleterious consequences on social behavior, anxiety, learning, memory, and/or repetitive behavior; phenotypes that are associated with ASD in humans [16,29]. In order to assess if there were any changes in sociability at six-week-old Pten ${ }^{Y 68 H /+}$ mice compared to Pten ${ }^{+/+}$ littermate controls, we employed the three-chamber test (Fig. 1g). We found that Pten ${ }^{\mathrm{Y} 68 \mathrm{H} /+}$ mice spent less time in the chamber containing the social target than the empty chamber $\left(\right.$ Mean $_{\Delta \text { Seconds }}=162 ; 95 \% \mathrm{Cl}: 121$ $-203 ; P<0.0001 ; \mathrm{N}_{+/+}=6 ; \mathrm{N}_{\mathrm{Y} 68 \mathrm{H} /+}=6 ;$ Fig. 1h). Then, utilizing the same three-chamber test model, we assessed changes in preference for social novelty by placing a familiar mouse in one chamber, and a novel social target in the other. We found a significant shift for Pten ${ }^{Y 68 H /+}$ mice toward a reduced preference for a novel versus familiar social target compared to $P$ ten $^{+/+}$littermate controls $\left(\right.$Mean $_{\triangle \mathrm{DSeconds}}=101 ; 95 \% \mathrm{Cl}$ : $56.5-145 ; P<0.0001 ; \mathrm{N}_{+/+}=6 ; \mathrm{N}_{\mathrm{Y} 68 \mathrm{H} /+}=6$; Fig. 1i). In order to assess repetitive behavior, since it is defined as one of the two core behavioral domains of ASD [42], we performed the marble burying test with six-weekold Pten ${ }^{Y 68 H /+}$ mice. We found that the cages of $P \operatorname{ten}^{Y 68 H /+}$ mice had more marbles buried compared to Pten $^{+/+}$controls (Median ${ }_{\Delta \text { Marbles }}=4.68 ; 97 \% \mathrm{Cl}: 2.12-7.23 ; P=0.002$ ) and increased displacement of the marbles from their original positions compared to the cages of wildtype mice as assessed by visual inspection (Fig. 1j, k). In addition, the appearance of bedding surface in the cages of Pten ${ }^{Y 68 H /+}$ mice was indicative of repetitive burying and digging behavior (Fig.1j). All together these behavioral data demonstrate that Pten ${ }^{Y 68 H /+}$ mice exhibit decreased sociability and increased repetitive behavior, fitting the two core behavioral domains of ASD.

\section{Microglial activation in the brains of $P t e n^{Y 68 H /+}$ mice}

It has been well established that disrupted Pten expression in mice can lead to increased cellular proliferation, white matter abnormality, astrogliosis, and microglial activation in vivo [16,23,30,31].

Therefore, we sought to determine if there were any clear cellular pathologies in the brains of six-month-old Pten ${ }^{Y 68 H /+}$ mice, using immunofluorescence staining and Western blotting for markers specific to neurons, oligodendrocytes, astrocytes, and microglia. To our surprise we did not observe any significant abnormalities in gross white matter nor in the populations of oligodendrocytes, astrocytes, or neurons with respect to proliferation or activity (Additional file 1: Fig S2A-H); however, there was a trend showing a slight decline in cell populations of oligodendrocytes and astrocytes in Pten ${ }^{\mathrm{Y} 68 \mathrm{H} /+}$ mice compared to Pten ${ }^{+/+}$ controls (Additional file 1: Fig S2B-D).

Despite the unexpected observations made regarding neurons, astrocytes, and oligodendrocytes in the cortex of six-month-old Pten ${ }^{Y 68 H /+}$ mice, we did observe clear microglial activation in the cortex. We stained microglia for ionized calcium binding adapter 1 (Iba1) in the cortex of six-month-old Pten ${ }^{Y 68 H /+}$ mice and 
observed increased Iba1-positive cells and morphological changes indicative of microglial activation, such as increased cell area (Fig. 2a, b). Thus, we quantified the cell area of individual microglia in $\mathrm{Pten}^{\mathrm{Y} 68 \mathrm{H} /+}$ in vivo and found a significant increase in the cell area of these microglia $\left(\right.$ Mean $_{\Delta \text { CellArea }}=0.29 ; 95 \% \mathrm{Cl}: 0.12$ $-0.46 ; P=0.002$; Fig. 2c). Moreover, we measured the integrated density of the lba1 stain and found it to

be significantly increased in $P t^{Y} n^{Y 68 H /+}$ compared to $P t e n^{+/+}$microglia $\left(\right.$Mean $_{\Delta \text { IntDensity }}=0.37 ; 95 \% \mathrm{Cl}: 0.22$ $-1.5 ; P=0.025 ;$ Fig. 2d).

Next, we validated $P \operatorname{ten}^{Y 68 H /+}$ microglial activation by quantifying microglia-specific proteins via Western blot. We found a significant increase in Iba1 $(P=0.029)$ and C1q $(P=0.029)$, a secreted complement component, expression; both proteins are only expressed by microglia in the CNS, implicated in synaptic pruning, and involved in phagosome formation (Fig. $2 \mathrm{f}, \mathrm{g}$ ). These data suggest not only that microglia are activated, but also that they may have increased phagocytic capabilities. Together, these data indicate that alterations in Pten localization and expression via the $P \operatorname{ten}^{Y 68 H}$ mutation leave astrocyte and oligodendrocyte populations unaffected while contributing to a microglial pathology.

\section{$\mathrm{Pten}^{\mathrm{Y68H/+}}$ microglia have increased phagocytic ability and efficiency in vitro}

To assess phagocytosis in $\mathrm{Pten}^{\mathrm{Y} 68 \mathrm{H} / \mathrm{+}}$ microglia, we performed a phagocytosis assay and found the total number of $P \operatorname{ten}^{\mathrm{Y} 68 \mathrm{H} /+}$ phagocytic microglia was significantly higher than the number of microglia isolated from $P_{t e n}{ }^{+/}$littermate controls $\left(\right.$Mean $_{\Delta}=0.16 ; 95 \% \mathrm{Cl}: 0.040-0.27 ; P=0.01 ;$ Fig. 2h, i). In addition, phagocytic Pten ${ }^{Y 68 H /+}$ microglia were able to engulf more fluorescent beads compared to Pten $^{+/+}$littermate controls (Mean ${ }_{\Delta}=3.2 ; 95 \% \mathrm{Cl}: 2.5-4.0 ; P=0.005 ;$ Fig. $2 \mathrm{j}$ ). These data not only demonstrate that Pten ${ }^{Y 68 H /+}$ microglia have increased phagocytic ability and efficiency compared to wildtype controls, but also that this pathology arises cell-autonomously as these cellular phenotypes are observed in primary microglial cultures devoid of external influences from neurons, oligodendrocytes, or astrocytes.

\section{$P_{t e n}{ }^{Y 68 H /+}$ mutants do not exhibit activation of Akt, Erk1/2, and $S 6$ in the cortex at six-months-of-age}

Next, we wished to determine if the changes in macrocephaly, behavior, microglial activation, and increased phagocytosis were associated with changes in downstream canonical Pten signaling, namely, $\mathrm{Pi} 3 \mathrm{k} / \mathrm{Akt} / \mathrm{mTor}$ activation. It has been previously reported and well established, including in other Pten mouse models, that disruptions of Pten expression and protein stability can lead to increased downstream phosphorylation and activation of S6, Akt, and Erk1/2 [17,20,23]. Therefore, we used six-month-old cortical lysates and performed Western blot analyses for Pten, Akt, P-Akt (S473), Erk1/2, P-Erk1/2, S6, and P-S6. We found a significant decrease in Pten expression, which is consistent with previous reports regarding human PTEN $^{Y 68 H}[33,34,43]$. However, we did not observe any significant differences in the phosphorylation of Akt, Erk1/2, or S6, though there was a trend toward increased phosphorylation of Akt and S6 (Additional file 1: Fig S3A-E). To our surprise, these data show that the pathologies found in $\mathrm{Pten}^{\mathrm{Y68H} /+}$ mutants are not necessarily associated with global changes in the classic signaling cascades downstream of Pten. Therefore, we sought to determine if nuclear-predominant Pten results in downstream transcriptome-wide changes, which might at least in part explain our behavior and cellular observations in vivo and in vitro. 


\section{Transcriptomic characterization of the $P t e n^{Y 68 H /+}$ cortex}

Given the surprising lack of disruption of canonical signaling downstream of Pten in the context of the striking behavioral and cellular findings, we performed a transcriptomic survey of the cortex of young adult (six-week-old or P40), male mice $\left(\mathrm{N}_{+/+}=6 ; \mathrm{N}_{\mathrm{Y} 68 \mathrm{H} /+}=5\right)$. RNA-sequencing analysis of cortical RNA identified 332 differentially expressed genes (threshold: $P<0.05 ; \log _{2}$ (Fold Change) $\geq 1.0$ or $\log _{2}($ Fold Change $) \leq$ -1.0; Additional file 2), which are summarized in a volcano plot (Fig. 3a). The volcano plot also illustrates a skew toward overexpression with relatively fewer under expressed genes being observed. Moreover, the changes in gene expression are visualized in a heatmap (threshold: $P<0.001$ ), showing a clear separation between genotypes with a general pattern of increased expression in the heterozygous mutant and decreased expression in the wildtype (Fig. 3b).

To gain insight into the biology affected by the expression changes observed in the $\mathrm{Pten}^{Y 68 \mathrm{H} /+}$ cortex, we performed Ingenuity Pathway Analysis (IPA), which identified the top "canonical pathways" that show enrichment beyond random chance based on the input gene list, i.e. the genes showing differential expression (threshold: $P<0.05 ; \log _{2}\left(\right.$ Fold Change) $\geq 1.0$ or $\log _{2}$ (Fold Change) $\leq-1.0$ ). The top ten pathways are all related to cellular stress and inflammation signaling (Fig. $3 \mathrm{c}$ ). This signature is driven by the differential expression of Card10, I/1r1, Ngfr, Tcf7/2, and Ttr, where I/1r1, the interleukin 1 receptor type 1 gene, appears in the associated lists of $90 \%$ of the pathways (Fig. $3 \mathrm{c}$ ). The top network showing how the differentially expressed molecules are biologically related implicates $T c f 7 / 2$ as an important regulatory node given that it has the highest degree centrality (i.e., 12) in the network (Fig 3d). Furthermore, using STRING analysis, an important gene-gene association network was identified from among the differentially expressed genes, implicating oxytocinergic signaling. Differential expression analysis found a roughly fivefold increase in oxytocin $(O x t)$, and network analysis of associated genes showing DE found a small network where $O x t$ has the highest degree and betweenness centrality (Fig 3e; Additional file 2).

Given the importance of oxytocin signaling to social behavior, we sought to understand more about the possible biological effects of increased expression of Oxt. Thus, we deployed IPA's Molecule Activity Predictor (MAP) to understand how the five-fold increase in Oxt may affect downstream interactors. From the top 10 molecules directly downstream of $O x t$, we found that increased $O x t$ expression predicts an increase in dopamine, calcium, Prkcz, and Egfr activity and a decrease in Crh and Fos activity (Fig. 3f). Finally, we sought to confirm the increase in Oxt expression in the brain of $\mathrm{Pten}^{\mathrm{Y} 68 \mathrm{H} /+}$ mice so we performed immunofluorescence staining for Oxt in six-week-old hypothalamus. By visual inspection alone, we found a dramatic increase in Oxt expression in the paraventricular neurons (PVN) of the hypothalamus of six-weekold Pten ${ }^{Y 68 \mathrm{H} /+}$ mice ( $\mathrm{N}=3$; Fig. $\left.4 a\right)$. We then quantified Oxt expression per individual PVN cell soma and found significantly increased Oxt expression in $\mathrm{Pten}^{\mathrm{Y} 68 \mathrm{H} /+}$ compared to wildtype controls $\left(\mathrm{Mean}_{\Delta \mathrm{IntDensity}}=\right.$ 6630; 95\% Cl: 4860 - 8400; $P<0.0001$; Fig 4b). In addition, we plotted the average global expression of Oxt per biological replicate to show these data were not skewed $\left(\right.$ Median $_{\Delta \ln \text { tDensity }}=7140 ; 97 \% \mathrm{Cl}: 3700-$ 10600; $P=0.0045 ;$ Fig. 4c). Given these data, it is clear nuclear-predominant Pten, $P \operatorname{ten}^{Y 68 H}$, associates with increased Oxt expression in the brain. 


\section{Discussion}

In this study, we demonstrate that the nuclear-predominant $P \operatorname{Pten}^{Y 68 \mathrm{H}}$ mutation in mice results in decreased social activity, decreased interest in novel social stimuli, increased perseverative behavior, increased microglial activation, and increased neural oxytocin levels. We show that in addition to deficits in social behavior, $P_{t e n^{Y 68 H /+}}$ mice have macrocephaly from increased brain mass (Fig. 1). Moreover, we show that $\mathrm{Pten}^{\mathrm{Y} 68 \mathrm{H} / \mathrm{+}}$ microglia are activated in vivo, expressing elevated amounts of $\mathrm{C} 1 \mathrm{q}$ and displaying enhanced phagocytic activity (Fig. 2). Despite clear organismal and cellular phenotypes, we found that $\mathrm{Pten}^{\mathrm{Y} 68 \mathrm{H} /+}$ brains do not exhibit disruption of canonical signaling downstream of Pten. Therefore, we resorted to interrogating the biology globally. As such, our transcriptomic survey of the cortex of $\mathrm{Pten}^{\mathrm{Y} 68 \mathrm{H} / \mathrm{t}}$ mice identified broad changes in gene expression much of which implicated neuroinflammatory or other neurological pathways, including the striking finding of increased oxytocin expression (Fig. 3). The oxytocin expression finding was confirmed at the protein level via staining of the hypothalamus (Fig. 4). Together these data implicate steady-state Pten levels as an important cell-autonomous regulator of microglial morphology and activity and suggest that a shift toward nuclear-predominant Pten expression provokes disruption of the oxytocin system in the brain.

Murine models of increasing specificity have established that Pten and related downstream signaling participates in the regulation of social behavior, CNS morphology, and neuronal and glial function $[23,25,44-61]$. Consistent behavioral phenotypes of altered social behavior persist when loss of Pten expression is restricted to mature neurons or neuronal precursors, and these models often have impaired learning/memory, increased anxiety, or altered activity/motor ability $[16,44,46,48]$. These behavioral abnormalities contrast somewhat with those observed in our germline Pten ${ }^{m 3 m 4}$, cytoplasmic-predominant Pten mislocalization model [23]. Pten ${ }^{m 3 m 4 / m 3 m 4}$ mice maintain relatively normal capacities for learning and memory, while showing a sex-specific (i.e., male) increase in social motivation and severely impaired motor coordination [16,23]. Pten ${ }^{Y 68 H /+}$ mice show decreased sociability and interest in social novelty with increased repetitive behavior, such as marble burying and nesting. It is difficult to assert confidently the sources of variability in the behavioral phenotypes observed in these various Pten mouse models; however, the Pten ${ }^{m 3 m 4 / m 3 m 4}$ and $P t e n^{Y 68 H /+}$ models are quite distinct from Pten knock-out or conditional knock-out models in that the mutations are designed to disrupt Pten localization instead of completely eliminating all Pten expression and thus functionality. Moreover, the Pten ${ }^{m 3 m 4}$ and $P t e n^{Y 68 H}$ mutations aim to mimic, or at least simulate, mutations observed in PTEN-ASD individuals, especially the latter mutation. In terms of the behavioral differences between the $P t e n^{m 3 m 4 / m 3 m 4}$ and $P t e n^{Y 68 H /+}$ models, it is also difficult to confidently ascribe certain behaviors to Pten localization partially because both mutations disrupt Pten stability and phosphatase activity to some extent; for instance, we know that the $P \operatorname{ten}^{\mathrm{Y} 68 \mathrm{H}}$ mutation likely has a very damaging effect on the stability of Pten [43]. However, it is worth noting that the cytoplasmic versus nuclear localization models appear to associate with contrasting effects on social motivation. It is likely that additional models and elegantly designed experiments will be needed to accurately associate Pten localization changes with specific behavioral changes in mice. 
Beyond the behavioral phenotypes, our contrasting Pten models do reveal something about the effects of Pten localization on glial phenotypes. The $P t e n^{m 3 m 4 / m 3 m 4}$ mouse has aggressive oligodendrocyte and astrocyte pathologies, including increased myelination [23,30], whereas the $\mathrm{Pten}^{\mathrm{Y} 68 \mathrm{H} / \mathrm{+}}$ mouse has no apparent oligodendrocyte or astrocyte pathologies (Additional file 1: Fig S2a-h). However, both the $\mathrm{Pten}^{\mathrm{m} 3 \mathrm{~m} 4 / \mathrm{m} 3 \mathrm{~m} 4}$ and $\mathrm{Pten}^{\mathrm{Y} 68 \mathrm{H} /{ }^{+}}$mice have activated microglia with enhanced phagocytic activity. Comparatively, these findings suggest that the localization affects glial phenotypes, where less nuclear Pten provokes oligodendrocyte and astrocyte pathologies. On the other hand, localization appears to not have an effect on microglia behavior, rather the total steady-state level of Pten appears to regulate microglia function. Both the Pten ${ }^{m 3 m 4}$ and $P \operatorname{Pten}^{Y 68 H}$ mutations disrupt Pten stability and both have activated microglia. Glia development and function appear to be tied closely to various aspects of Pten activity, presenting a likely fruitful area of future study.

Possibly the most surprising and important finding of this study is the observation of the lack of disruption of downstream canonical signaling, but instead, increased oxytocin expression in the $\mathrm{Pten}^{Y 68 \mathrm{H} /{ }^{+} \text {brain, }}$ which occurs in a model with clear behavioral abnormalities. A wealth of research, including extensive animal modeling, demonstrates persuasively that the oxytocin system is important to prosocial cognition [62]. In fact, research has shown that oxytocin may be a viable therapeutic modality for ameliorating social deficits in individuals with ASD [62-65]. Interestingly, many of the models referenced above (e.g., Oxt knockouts) have deficits in the oxytocin system where mice either have lower circulating Oxt or an inability to respond to exogenous Oxt due to knockout (i.e. oxytocin receptor knockout). Our molecular phenotype for oxytocin is quite the opposite. This may seem paradoxical that a mouse with behavioral deficits has an elevated amount of a prosocial neuropeptide. However, it has been shown that high exogenous doses of oxytocin can paradoxically provoke an anxiogenic response due to the excess oxytocin, after oxytocin receptor (OXTR) saturation, acting on vasopressin receptors $[65,66]$. Moreover, it has also been observed in other models, such as the BTBR mouse, that increased oxytocin expression and social deficits (i.e. increased anxiety) can co-occur [67]. Subsequently, we believe that the increased Oxt expression observed in the RNA-sequencing experiment (Fig. 3) and validated with staining of the PVN (Fig. 4) likely accounts for some of the behavioral abnormalities observed in the $\mathrm{Pten}^{\mathrm{Y} 68 \mathrm{H} /+}$ mouse. This is important as this is the first time the oxytocin system has be observed as perturbed in a Pten model. Disruption of the oxytocin system may be specific to only a subset of Pten mutations, such as those that cause nuclear mislocalization. More research is required to sort out whether oxytocin system problems are common to all Pten models or only to a specific subset of mutations.

\section{Limitations}

As suggested above the strengths of our study are founded on the specificity of the mouse modeling (i.e., a knockin mutation identical to that observed in a PTEN-ASD individual) and the rigorous behavioral, cellular, and molecular phenotyping. However, there are limitations to our $P \operatorname{ten}^{\mathrm{Y} 68 \mathrm{H} /+}$ model, such as the deleterious effect of the $\mathrm{Y} 68 \mathrm{H}$ mutation on the stability of Pten $[34,43]$. The decreased stability of $\mathrm{Pten}^{\mathrm{Y} 68 \mathrm{H}}$ makes it difficult to absolutely attribute causality of any phenotype to the localization changes observed. Moreover, the localization changes themselves are not absolute either. As clearly shown in Fig. 1b and c, the change in 
localization is relative, and there is still plenty Ptenin the cytoplasm; though, the extent to which the cytoplasmic Pten is of wildtype or mutant providence is unknown as the germline nature of the model since the $P t e n^{Y 68 H / Y 68 H}$ genotype exhibits embryonic lethality, therefore requiring the study of only heterozygous mutants. It is unclear if the $\mathrm{Y} 68 \mathrm{H}$ mutation can function as a dominant negative mutant as has been described for other PTEN missense mutations [68], but there is some existing evidence to suggest that it does not function as such $[28,43,69]$. Future work designed to specifically interrogate questions about the functional effects of Pten localization on social and neurobiological phenotypes will have to utilize more extensive modeling strategies. However, the $\mathrm{Pten}^{\mathrm{Y} 68 \mathrm{H} /+}$ model is extremely useful for gaining important insights into these challenging and important scientific questions about pathophysiology, which should help inform monogenic ASD risk cases.

\section{Conclusion}

Although there, of course, remains much to explore about the $\mathrm{Pten}^{\mathrm{Y} 68 \mathrm{H} /+}$ mouse and other Pten models, especially in terms of the effects on social cognition and neurobiology, this study is an important step toward understanding how Pten localization in the brain can affect social cognition and neuronal and glial function. Until this study, it was unclear if decreased cytoplasmic expression of Pten would affect the CNS in such a dramatic fashion. Our work on the $\mathrm{Pten}^{\mathrm{Y} 68 \mathrm{H} /+}$ mouse demonstrates that social behavior can be modulated by mutations that shift Pten to the cytoplasm or nucleus, but the exact social phenotypes can be quite distinct. Contrastingly, certain glial phenotypes seem to be in part dependent on Pten localization as the $P t n^{Y 68 H /+}$ mouse shows no apparent changes in oligodendrocytes and astrocytes (Additional file 1: Fig S2), whereas its complement, the Pten ${ }^{m 3 m 4}$ model, show aggressive glial pathologies [23,30]. However, microglial dysfunction seems to be entirely independent of Pten localization, instead resulting from decreased Pten expression levels in general. In addition, we find prominent oxytocin overexpression in the hypothalamus of $\mathrm{Pten}^{\mathrm{Y} 68 \mathrm{H} /+}$ mice, thus linking Pten mutation and the oxytocin system for the first time. Finally, an interesting and potentially significant finding is that all of the molecular and cellular pathologies reported in this study appear to be independent of Pten canonical signaling at least at the cortical level in fairly homeostatic conditions (Additional file 1: Fig S3). In sum, this study demonstrates the importance of nuclear Pten to CNS morphology and function while linking Pten, a prominent ASD risk gene, to a neuroendocrine modulator of social behavior, oxytocin, in a murine model with clear social deficits.

\section{Abbreviations}

ASD: autism spectrum disorder; central nervous system: CNS; oxytocin: OXT; PTEN: Phosphatase and Tensin Homolog; PTEN Hamartoma Tumor Syndrome: PHTS

\section{Declarations}

\section{Acknowledgements}


We are grateful to Qi Yu for helping with mouse breeding and husbandry in the Eng lab, and Peter Bazely for bioinformatics core support. CE is the Sondra J. and Stephen R. Hardis Endowed Chair of Cancer Genomic Medicine at the Cleveland Clinic, and is an ACS Clinical Research Professor.

\section{Authors' contributions}

Conception and design: NS, ST, HP, CE

Experimentation and data acquisition: NS, ST, HP

Interpretation of data: NS, ST, HP, CE

Drafting of manuscript: ST, NS

Critical revisions of manuscript: ST, NS, CE

Final approval of manuscript: All authors

\section{Funding}

This study was funded, in part, by the Ambrose Monell Foundation and the Zacconi Program of PTEN Research Excellence.

\section{Availability of data and materials}

The datasets for this study are available from the corresponding author on reasonable request.

\section{Ethics approval}

This study was approved by Cleveland Clinic Lerner Research Institute's IACUC.

\section{Consent for publication}

Not applicable.

\section{Competing interests}

The authors declare that they have no competing interests.

\section{References}

1. Butler M, Dasouki M, Zhou X, Talebizadeh Z, Brown M, Takahashi T, et al. Subset of individuals with autism spectrum disorders and extreme macrocephaly associated with germline PTEN tumour suppressor gene mutations. J Med Genet. 2005 Apr;42(4):318-21.

2. Buxbaum JD, Cai G, Chaste P, Nygren G, Goldsmith J, Reichert J, et al. Mutation screening of the PTEN gene in patients with autism spectrum disorders and macrocephaly. Am J Med Genet Part B 
Neuropsychiatr Genet Off Publ Int Soc Psychiatr Genet. 2007 Jun 5;144B(4):484-91.

3. Varga EA, Pastore M, Prior T, Herman GE, McBride KL. The prevalence of PTEN mutations in a clinical pediatric cohort with autism spectrum disorders, developmental delay, and macrocephaly. Genet Med Off J Am Coll Med Genet. 2009 Feb;11(2):111-7.

4. Orrico A, Galli L, Buoni S, Orsi A, Vonella G, Sorrentino V. Novel PTEN mutations in neurodevelopmental disorders and macrocephaly. Clin Genet. 2009 Feb;75(2):195-8.

5. McBride KL, Varga EA, Pastore MT, Prior TW, Manickam K, Atkin JF, et al. Confirmation study of PTEN mutations among individuals with autism or developmental delays/mental retardation and macrocephaly. Autism Res Off J Int Soc Autism Res. 2010 Jun;3(3):137-41.

6. O’Roak BJ, Deriziotis P, Lee C, Vives L, Schwartz JJ, Girirajan S, et al. Exome sequencing in sporadic autism spectrum disorders identifies severe de novo mutations. Nat Genet. 2011 Jun;43(6):585-9.

7. O’Roak BJ, Vives L, Fu W, Egertson JD, Stanaway IB, Phelps IG, et al. Multiplex targeted sequencing identifies recurrently mutated genes in autism spectrum disorders. Science. 2012 Dec 21;338(6114):1619-22.

8. O’Roak BJ, Vives L, Girirajan S, Karakoc E, Krumm N, Coe BP, et al. Sporadic autism exomes reveal a highly interconnected protein network of de novo mutations. Nature. 2012 Apr 4;485(7397):246-50.

9. Klein S, Sharifi-Hannauer P, Martinez-Agosto JA. Macrocephaly as a clinical indicator of genetic subtypes in autism. Autism Res Off J Int Soc Autism Res. 2013 Feb;6(1):51-6.

10. De Rubeis S, He X, Goldberg AP, Poultney CS, Samocha K, Cicek AE, et al. Synaptic, transcriptional and chromatin genes disrupted in autism. Nature. 2014 Nov 13;515(7526):209-15.

11. Hobert JA, Embacher R, Mester JL, Frazier TW, Eng C. Biochemical screening and PTEN mutation analysis in individuals with autism spectrum disorders and macrocephaly. Eur J Hum Genet EJHG. 2014 Feb;22(2):273-6.

12. Marchese M, Conti V, Valvo G, Moro F, Muratori F, Tancredi R, et al. Autism-epilepsy phenotype with macrocephaly suggests PTEN, but not GLIALCAM, genetic screening. BMC Med Genet. 2014 Feb 27; $15: 26$.

13. Satterstrom FK, Kosmicki JA, Wang J, Breen MS, De Rubeis S, An J-Y, et al. Large-Scale Exome Sequencing Study Implicates Both Developmental and Functional Changes in the Neurobiology of Autism. Cell. 2020 06;180(3):568-584.e23.

14. Fombonne E, Rogé B, Claverie J, Courty S, Frémolle J. Microcephaly and macrocephaly in autism. J Autism Dev Disord. 1999 Apr;29(2):113-9.

15. O’Roak BJ, Vives L, Girirajan S, Karakoc E, Krumm N, Coe BP, et al. Sporadic autism exomes reveal a highly interconnected protein network of de novo mutations. Nature. 2012 Apr 4;485(7397):246-50.

16. Tilot AK, Frazier TW, Eng C. Balancing Proliferation and Connectivity in PTEN-associated Autism Spectrum Disorder. Neurother J Am Soc Exp Neurother. 2015 Jul;12(3):609-19.

17. Yehia L, Ngeow J, Eng C. PTEN-opathies: from biological insights to evidence-based precision medicine. J Clin Invest. 2019 Feb 1;129(2):452-64. 
18. Marsh DJ, Kum JB, Lunetta KL, Bennett MJ, Gorlin RJ, Ahmed SF, et al. PTEN mutation spectrum and genotype-phenotype correlations in Bannayan-Riley-Ruvalcaba syndrome suggest a single entity with Cowden syndrome. Hum Mol Genet. 1999 Aug;8(8):1461-72.

19. Maehama T, Dixon JE. The tumor suppressor, PTEN/MMAC1, dephosphorylates the lipid second messenger, phosphatidylinositol 3,4,5-trisphosphate. J Biol Chem. 1998 May 29;273(22):13375-8.

20. Worby CA, Dixon JE. PTEN. Annu Rev Biochem. 2014;83:641-69.

21. Hasle N, Matreyek KA, Fowler DM. The Impact of Genetic Variants on PTEN Molecular Functions and Cellular Phenotypes. Cold Spring Harb Perspect Med. 2019 01;9(11).

22. Ho J, Cruise ES, Dowling RJO, Stambolic V. PTEN Nuclear Functions. Cold Spring Harb Perspect Med. 2020 May 1;10(5).

23. Tilot AK, Gaugler MK, Yu Q, Romigh T, Yu W, Miller RH, et al. Germline disruption of Pten localization causes enhanced sex-dependent social motivation and increased glial production. Hum Mol Genet. 2014 Jun 15;23(12):3212-27.

24. Tilot AK, Bebek G, Niazi F, Altemus J, Romigh T, Frazier TW, et al. Neural transcriptome of constitutional Pten dysfunction in mice and its relevance to human idiopathic Autism Spectrum Disorder. Mol Psychiatry. 2016 Jan;21(1):118-25.

25. Fricano-Kugler CJ, Getz SA, Williams MR, Zurawel AA, DeSpenza T, Frazel PW, et al. Nuclear Excluded Autism-Associated Phosphatase and Tensin Homolog Mutations Dysregulate Neuronal Growth. Biol Psychiatry. 2018 15;84(4):265-77.

26. Frazier TW, Embacher R, Tilot AK, Koenig K, Mester J, Eng C. Molecular and phenotypic abnormalities in individuals with germline heterozygous PTEN mutations and autism. Mol Psychiatry. 2015 Sep;20(9):1132-8.

27. Leslie NR, Longy M. Inherited PTEN mutations and the prediction of phenotype. Semin Cell Dev Biol. 2016 Apr 1;52:30-8.

28. Mighell TL, Thacker S, Fombonne E, Eng C, O’Roak BJ. An Integrated Deep-Mutational-Scanning Approach Provides Clinical Insights on PTEN Genotype-Phenotype Relationships. Am J Hum Genet. 2020 04;106(6):818-29.

29. Yehia L, Keel E, Eng C. The Clinical Spectrum of PTEN Mutations. Annu Rev Med. 2020;71(1):103-16.

30. Lee H, Thacker S, Sarn N, Dutta R, Eng C. Constitutional mislocalization of Pten drives precocious maturation in oligodendrocytes and aberrant myelination in model of autism spectrum disorder. Transl Psychiatry. 2019 Jan 17;9(1):13.

31. Sarn N, Jaini R, Thacker S, Lee H, Dutta R, Eng C. Cytoplasmic-predominant Pten increases microglial activation and synaptic pruning in a murine model with autism-like phenotype. Mol Psychiatry. 2020 Feb 13;

32. Kang SC, Jaini R, Hitomi M, Lee H, Sarn N, Thacker S, et al. Decreased nuclear Pten in neural stem cells contributes to deficits in neuronal maturation. Mol Autism. 2020 Jun 1;11(1):43.

33. Lobo GP, Waite KA, Planchon SM, Romigh T, Nassif NT, Eng C. Germline and somatic cancer-associated mutations in the ATP-binding motifs of PTEN influence its subcellular localization and tumor 
suppressive function. Hum Mol Genet. 2009 Aug 1;18(15):2851-62.

34. He X, Ni Y, Wang Y, Romigh T, Eng C. Naturally occurring germline and tumor-associated mutations within the ATP-binding motifs of PTEN lead to oxidative damage of DNA associated with decreased nuclear p53. Hum Mol Genet. 2011 Jan 1;20(1):80-9.

35. Yang M, Silverman JL, Crawley JN. Automated three-chambered social approach task for mice. Curr Protoc Neurosci. 2011 Jul;Chapter 8:Unit 8.26.

36. Angoa-Pérez M, Kane MJ, Briggs DI, Francescutti DM, Kuhn DM. Marble burying and nestlet shredding as tests of repetitive, compulsive-like behaviors in mice. J Vis Exp JoVE. 2013 Dec 24;(82):50978.

37. Dobin A, Gingeras TR. Mapping RNA-seq Reads with STAR. Curr Protoc Bioinforma. 2015 Sep 3;51:11.14.1-11.14.19.

38. Dobin A, Gingeras TR. Optimizing RNA-Seq Mapping with STAR. Methods Mol Biol Clifton NJ. 2016;1415:245-62.

39. Dobin A, Davis CA, Schlesinger F, Drenkow J, Zaleski C, Jha S, et al. STAR: ultrafast universal RNA-seq aligner. Bioinforma Oxf Engl. 2013 Jan 1;29(1):15-21.

40. Mester JL, Tilot AK, Rybicki LA, Frazier TW, Eng C. Analysis of prevalence and degree of macrocephaly in patients with germline PTEN mutations and of brain weight in Pten knock-in murine model. Eur $\mathrm{J}$ Hum Genet EJHG. 2011 Jul;19(7):763-8.

41. Tan M-H, Mester J, Peterson C, Yang Y, Chen J-L, Rybicki LA, et al. A Clinical Scoring System for Selection of Patients for PTEN Mutation Testing Is Proposed on the Basis of a Prospective Study of 3042 Probands. Am J Hum Genet. 2011 Jan 7;88(1):42-56.

42. Association AP. Diagnostic and Statistical Manual of Mental Disorders (DSM-5®). American Psychiatric Pub; 2013. 1414 p.

43. Matreyek KA, Starita LM, Stephany JJ, Martin B, Chiasson MA, Gray VE, et al. Multiplex assessment of protein variant abundance by massively parallel sequencing. Nat Genet. 2018 Jun;50(6):874.

44. Kwon C-H, Zhou J, Li Y, Kim KW, Hensley LL, Baker SJ, et al. Neuron-specific enolase-cre mouse line with cre activity in specific neuronal populations. Genes N Y N 2000. 2006 Mar;44(3):130-5.

45. Ogawa S, Kwon C-H, Zhou J, Koovakkattu D, Parada LF, Sinton CM. A seizure-prone phenotype is associated with altered free-running rhythm in Pten mutant mice. Brain Res. 2007 Sep 7;1168:112-23.

46. Zhou J, Blundell J, Ogawa S, Kwon C-H, Zhang W, Sinton C, et al. Pharmacological inhibition of mTORC1 suppresses anatomical, cellular, and behavioral abnormalities in neural-specific Pten knockout mice. J Neurosci Off J Soc Neurosci. 2009 Feb 11;29(6):1773-83.

47. Page DT, Kuti OJ, Prestia C, Sur M. Haploinsufficiency for Pten and Serotonin transporter cooperatively influences brain size and social behavior. Proc Natl Acad Sci U S A. 2009 Feb 10;106(6):1989-94.

48. Amiri A, Cho W, Zhou J, Birnbaum SG, Sinton CM, McKay RM, et al. Pten deletion in adult hippocampal neural stem/progenitor cells causes cellular abnormalities and alters neurogenesis. J Neurosci Off $J$ Soc Neurosci. 2012 Apr 25;32(17):5880-90.

49. Kazdoba TM, Sunnen CN, Crowell B, Lee GH, Anderson AE, D’Arcangelo G. Development and characterization of NEX-Pten, a novel forebrain excitatory neuron-specific knockout mouse. Dev 
Neurosci. 2012;34(2-3):198-209.

50. Clipperton-Allen AE, Page DT. Pten haploinsufficient mice show broad brain overgrowth but selective impairments in autism-relevant behavioral tests. Hum Mol Genet. 2014 Jul 1;23(13):3490-505.

51. Clipperton-Allen AE, Chen Y, Page DT. Autism-relevant behaviors are minimally impacted by conditional deletion of Pten in oxytocinergic neurons. Autism Res Off J Int Soc Autism Res. 2016;9(12):1248-62.

52. Lugo JN, Smith GD, Morrison JB, White J. Deletion of PTEN produces deficits in conditioned fear and increases fragile X mental retardation protein. Learn Mem Cold Spring Harb N. 2013 Nov 15;20(12):670-3.

53. Lugo JN, Smith GD, Arbuckle EP, White J, Holley AJ, Floruta CM, et al. Deletion of PTEN produces autism-like behavioral deficits and alterations in synaptic proteins. Front Mol Neurosci. 2014;7:27.

54. Chen Y, Huang W-C, Séjourné J, Clipperton-Allen AE, Page DT. Pten Mutations Alter Brain Growth Trajectory and Allocation of Cell Types through Elevated $\beta$-Catenin Signaling. J Neurosci Off J Soc Neurosci. 2015 Jul 15;35(28):10252-67.

55. Clipperton-Allen AE, Page DT. Decreased aggression and increased repetitive behavior in Pten haploinsufficient mice. Genes Brain Behav. 2015 Feb;14(2):145-57.

56. Igarashi A, Itoh K, Yamada T, Adachi Y, Kato T, Murata D, et al. Nuclear PTEN deficiency causes microcephaly with decreased neuronal soma size and increased seizure susceptibility. J Biol Chem. 2018 15;293(24):9292-300.

57. Chen C-J, Sgritta M, Mays J, Zhou H, Lucero R, Park J, et al. Therapeutic inhibition of mTORC2 rescues the behavioral and neurophysiological abnormalities associated with Pten-deficiency. Nat Med. 2019;25(11):1684-90.

58. Clipperton-Allen AE, Cohen OS, Aceti M, Zucca A, Levy J, Ellegood J, et al. Pten haploinsufficiency disrupts scaling across brain areas during development in mice. Transl Psychiatry [Internet]. Available from: http://feeds.nature.com/ r/tp/rss/current/ 3/TaNbTgrnJSQ/s41398-019-0656-6?

utm_source=researcher_app\&utm_medium=referral\&utm_campaign=RESR_MRKT_Researcher_inbound

59. Skelton PD, Frazel PW, Lee D, Suh H, Luikart BW. Pten loss results in inappropriate excitatory connectivity. Mol Psychiatry [Internet]. 2019 Apr 9 [cited 2019 Apr 15]; Available from: http://www.nature.com/articles/s41380-019-0412-6

60. Skelton PD, Stan RV, Luikart BW. The Role of PTEN in Neurodevelopment. Mol Neuropsychiatry. 2020 Apr;5(Suppl 1):60-71.

61. Skelton PD, Poquerusse J, Salinaro JR, Li M, Luikart BW. Activity-dependent dendritic elaboration requires Pten. Neurobiol Dis. 2020;134:104703.

62. Modi ME, Young LJ. The oxytocin system in drug discovery for autism: Animal models and novel therapeutic strategies. Horm Behav. 2012 Mar 1;61(3):340-50.

63. Andari E, Duhamel J-R, Zalla T, Herbrecht E, Leboyer M, Sirigu A. Promoting social behavior with oxytocin in high-functioning autism spectrum disorders. Proc Natl Acad Sci. 2010 Mar 2;107(9):438994. 
64. Preckel K, Kanske P, Singer T, Paulus FM, Krach S. Clinical trial of modulatory effects of oxytocin treatment on higher-order social cognition in autism spectrum disorder: a randomized, placebocontrolled, double-blind and crossover trial. BMC Psychiatry. 2016 Sep 21;16(1):329.

65. Quintana DS, Westlye LT, Hope S, Nærland T, Elvsåshagen T, Dørum E, et al. Dose-dependent socialcognitive effects of intranasal oxytocin delivered with novel Breath Powered device in adults with autism spectrum disorder: a randomized placebo-controlled double-blind crossover trial. Transl Psychiatry. 2017 May;7(5):e1136-e1136.

66. Preckel K, Kanske P. Amygdala and oxytocin functioning as keys to understanding and treating autism: Commentary on an RDoC based approach. Neurosci Biobehav Rev. 2018 Nov 1;94:45-8.

67. Silverman JL, Yang M, Turner SM, Katz AM, Bell DB, Koenig JI, et al. Low stress reactivity and neuroendocrine factors in the BTBR T+tf/J mouse model of autism. Neuroscience. $2010 \mathrm{Dec}$ 29;171(4):1197-208.

68. Papa A, Wan L, Bonora M, Salmena L, Song MS, Hobbs RM, et al. Cancer-associated PTEN mutants act in a dominant negative manner to suppress PTEN protein function. Cell. 2014 Apr 24;157(3):595-610.

69. Mighell TL, Evans-Dutson S, O’Roak BJ. A Saturation Mutagenesis Approach to Understanding PTEN Lipid Phosphatase Activity and Genotype-Phenotype Relationships. Am J Hum Genet. 2018 May 3;102(5):943-55.

\section{Figures}



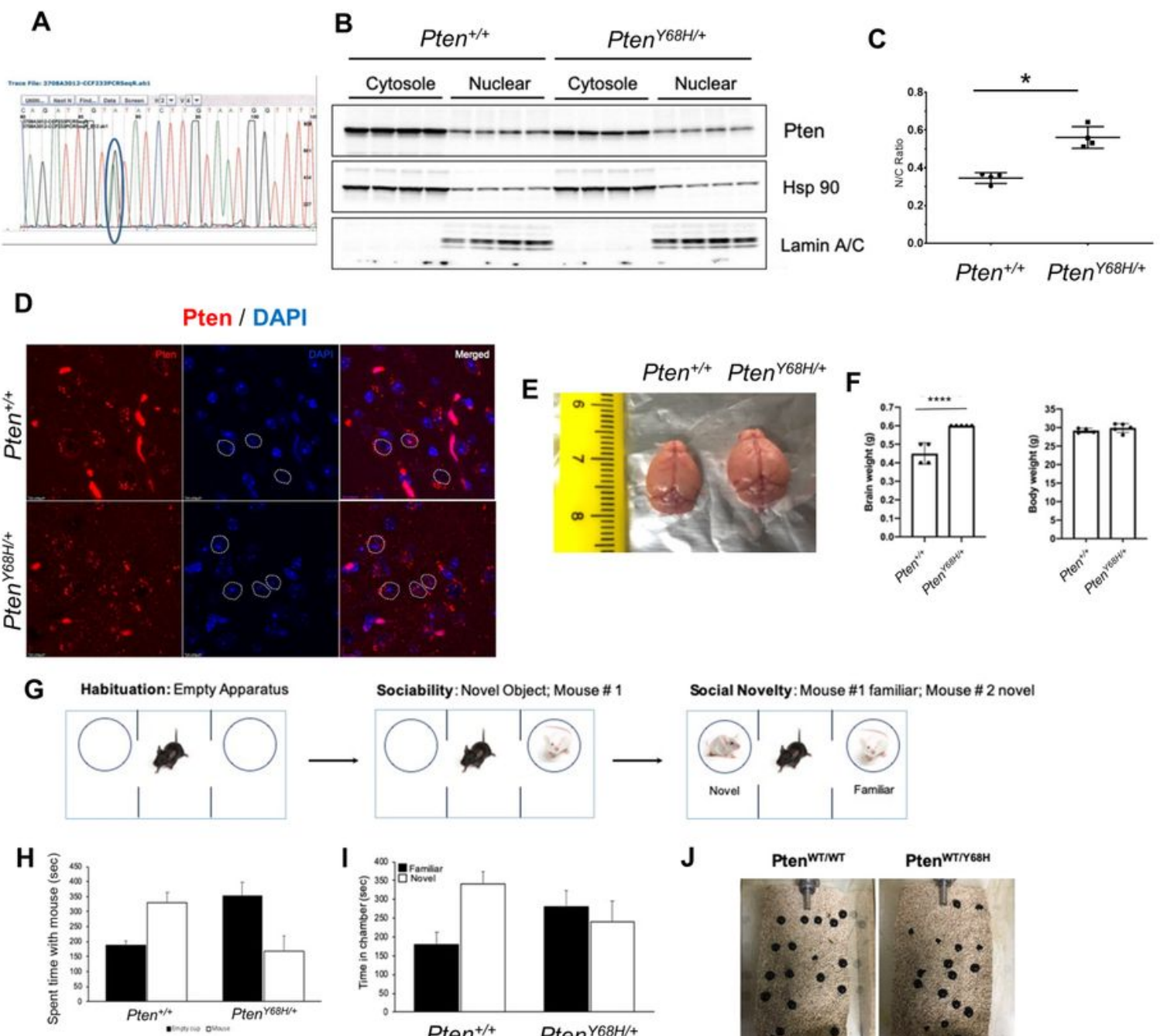

K
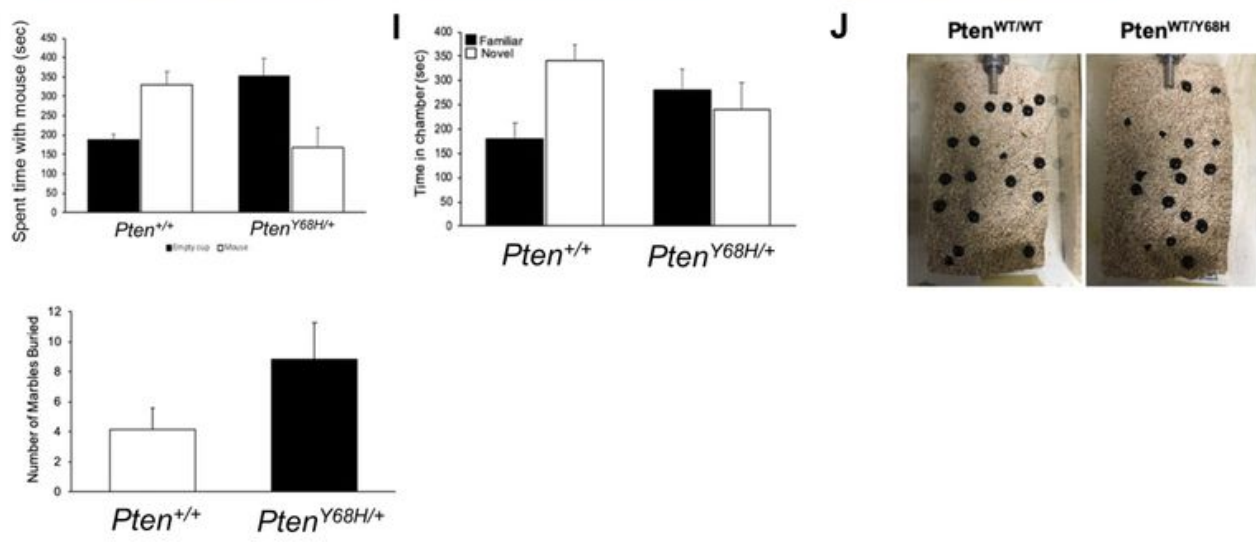

\section{Figure 1}

Gross morphological and behavioral phenotyping of Pten $\mathrm{Y} 68 \mathrm{H} /+$ mice, showing macrocephaly, social impairment, and perseverative behavior. a Visualization of single missense mutation in exon 3 of mouse Pten c.202 $\mathrm{T}>\mathrm{C}$, converting tyrosine $(\mathrm{Y})$ residue 68 into histidine $(\mathrm{H})$, which was subsequently used to generate the germline Pten $\mathrm{Y} 68 \mathrm{H} /+$ mouse model. b Nuclear-cytoplasmic fractionation performed on sixmonth-old Pten+/+ and PtenY68H/+ mouse cortical tissue and visualized via Western blot, targeting Pten as well as Hsp90 (cytoplasmic), and Lamin A/C (nuclear) to demonstrate fraction purity. c Quantification of 
nuclear-cytoplasmic Pten ratio from Western blot in panel b (Median $\Delta$ N/CRatio $=0.20,97 \%$ Cl: $0.14-0.34$, $P=0.029$ ). $d$ Immunofluorescence staining of six-month-old mouse cortex, visualizing Pten (red) and DAPI (blue) in neurons of Pten+/+ and Pten $\mathrm{Y} 68 \mathrm{H} /+$ mice. e Representative gross anatomical image, showing brain volume and morphology of six-month-old Pten+/+ and PtenY68H/+mice. f Quantification of total brain mass and body weight in grams $(\mathrm{g})$ of six-month-old Pten+/+and PtenY $68 \mathrm{H} /+$ (median $\Delta \mathrm{BrainMass}=$ $0.15,97 \% \mathrm{Cl}: 0.090-0.21, \mathrm{P}<0.0001)$. g Schematic of the experimental design for the three-chamber sociability and social novelty tests as they were performed on six-week-old Pten $+/+$ and Pten $\mathrm{Y} 68 \mathrm{H} /+$ mice. $\mathrm{h}$ Quantification of the three-chamber sociability test performed with six-week-old Pten+/+ and PtenY68H/+ mice, showing time spent in empty chamber or in chamber containing the social target (Mean $\Delta$ Seconds $=$ 162; $95 \% \mathrm{Cl}: 121-203 ; \mathrm{P}<0.0001 ; \mathrm{N}+/+=6 ; \mathrm{NY} 68 \mathrm{H} /+=6$ ). i Quantification of the three-chamber social novelty test performed with six-week-old Pten $+/+$ and Pten $\mathrm{Y} 68 \mathrm{H} /+$ mice, showing time spent in chamber containing familiar or novel social target (Mean $\Delta$ Seconds $=101 ; 95 \% \mathrm{Cl}: 56.5-145 ; \mathrm{P}<0.0001 ; \mathrm{N}+/+=6$; $\mathrm{NY} 68 \mathrm{H} /+=6$ ). $\mathrm{j}$ Representative image of marble burring test results for Pten $+/+$ and PtenY68H/+ mice. $\mathrm{k}$ Quantification of marble burying test, showing mean marbles buried between Pten $+/+$ and $\mathrm{Pten} \mathrm{Y} 68 \mathrm{H} /+$ mice (Median $\Delta$ Marbles $=4.68 ; 97 \% \mathrm{Cl}: 2.12-7.23 ; \mathrm{P}=0.002)$. P-value key: ${ }^{*} \mathrm{P}<0.05$ and ${ }^{\star \star \star \star} \mathrm{P}<0.0001$. 

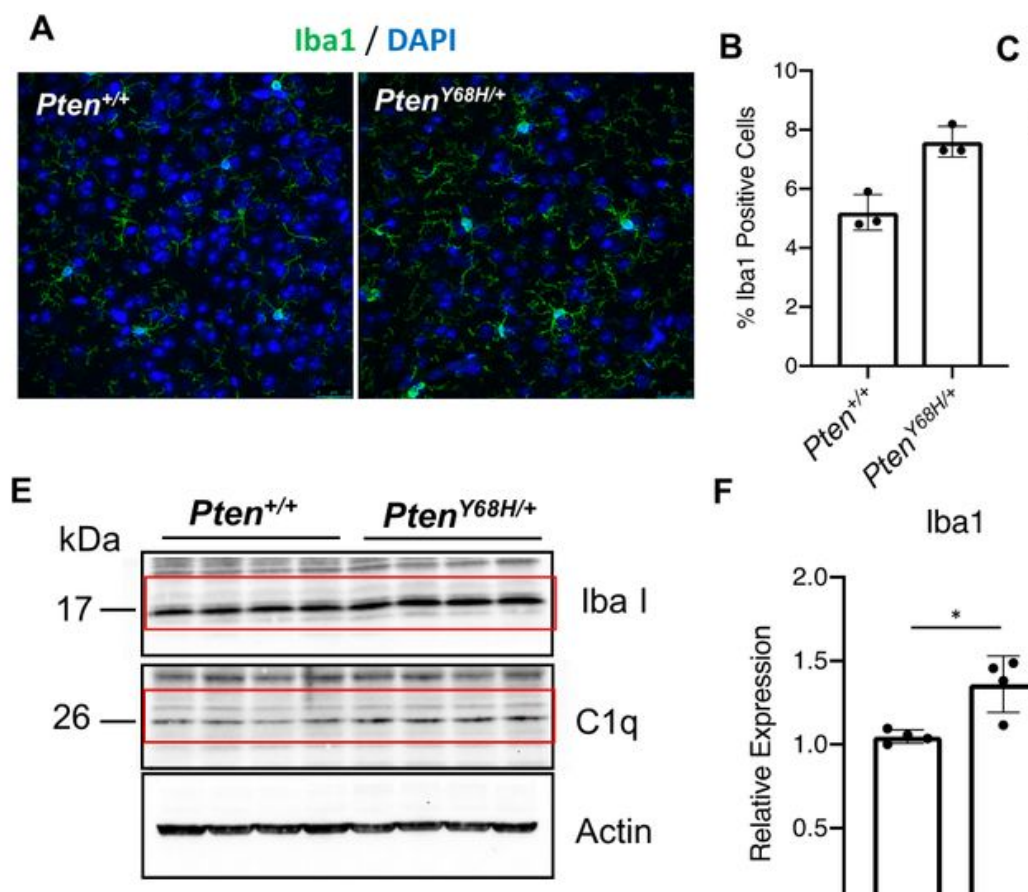

F lba1

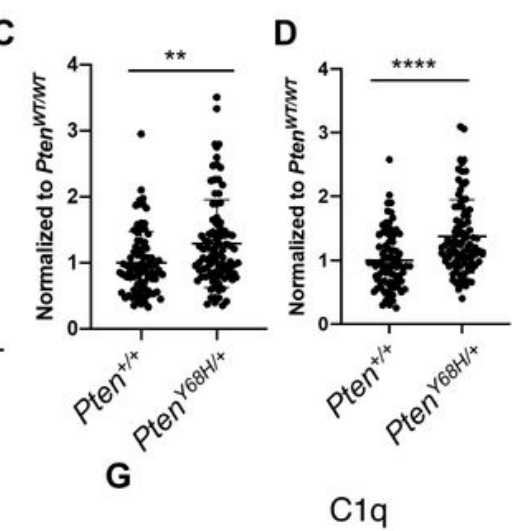

H
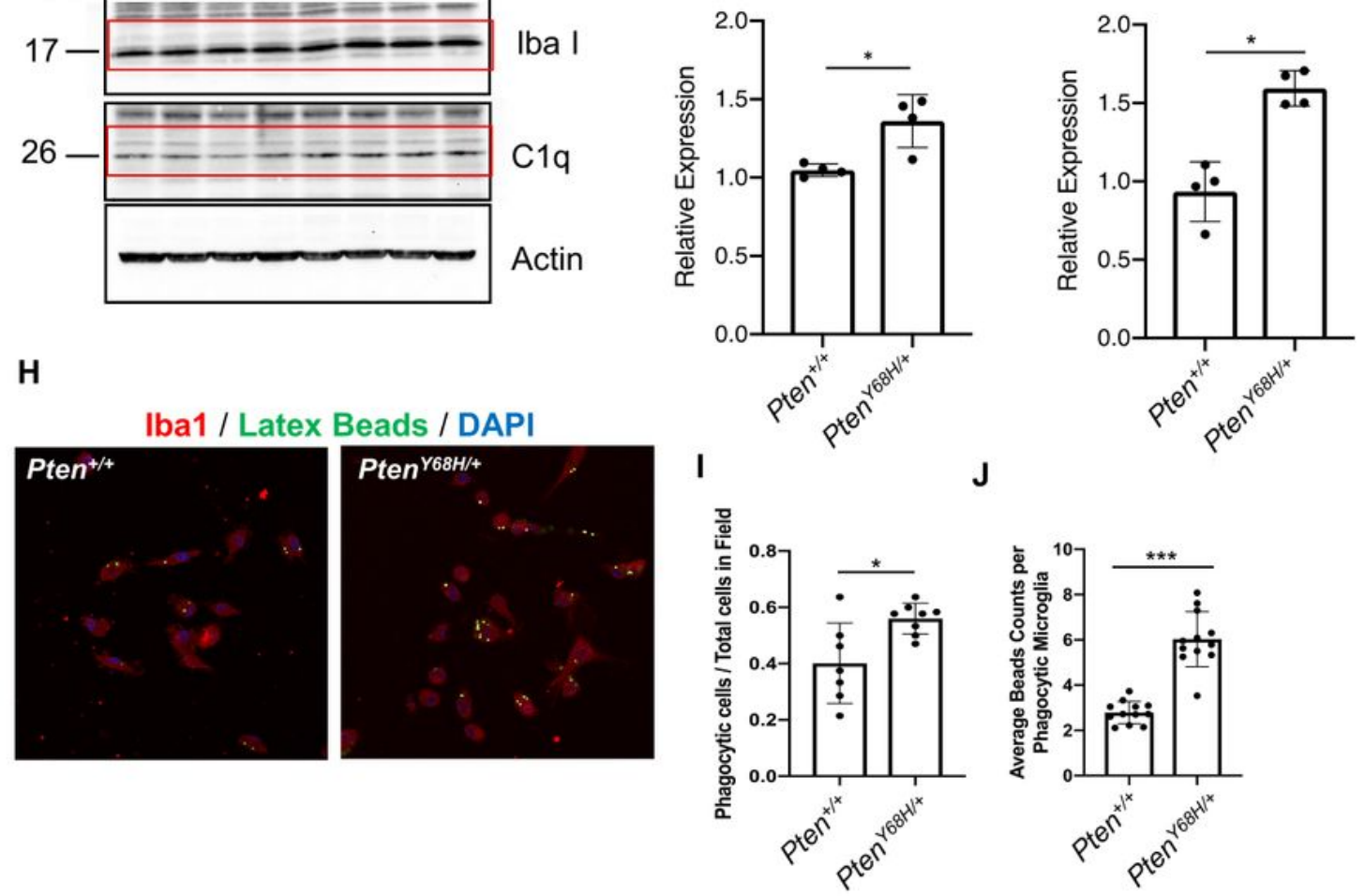

\section{Figure 2}

Evidence for a microglial pathology in the Pten $\mathrm{Y} 68 \mathrm{H} /+$ cortex. a Representative immunofluorescence staining of six-month-old Pten+/+ and PtenWT/Y68H cortical microglia with Iba1 (green) and DAPI (blue). b Percentage of microglia counted in six-month-old Pten+/+ and PtenY68H/+ cortex $(\mathrm{N}=3)$; total Iba1positive cells normalized to total DAPI-positive cells from panel a experiments. $c$ and d Quantification of individual microglial cell area (Mean $\Delta$ CellArea $=0.29 ; 95 \% \mathrm{Cl}$ : $0.12-0.46 ; \mathrm{P}=0.002$ ) and integrated density (Mean $\Delta$ IntDensity $=0.37 ; 95 \% \mathrm{Cl}: 0.22-1.5 ; \mathrm{P}=0.025)$ of lba1 from six-month-old Pten+/+and 
Pten $\mathrm{Y} 68 \mathrm{H} /+$ as captured in panel a experiments. e Western blot of six-month-old Pten+/+ and PtenY68H/+ cortical lysates, probing Iba1, C1q, and Actin. $f$ and $g$ Quantification of Iba1 and C1q expression normalized to Actin from Western blot in panel d, finding a significant increase in both $(P=0.029)$. h Representative image from phagocytosis assay, showing increased fluorescent bead (green) engulfment by primary microglia labeled with Iba1 (red), which were isolated from P2 Pten+/+ and PtenY68H/+ pups. i and j Quantification of phagocytosis assay experiments (panel $\mathrm{h}$ ), including the number of phagocytic cells/total number of cells in the field (Mean $\Delta=0.16 ; 95 \% \mathrm{Cl}: 0.04-0.27, \mathrm{P}=0.01$ ) and the average number of fluorescent beads counted in phagocytic cells (Mean $\Delta=3.24 ; 95 \% \mathrm{Cl}: 2.5-4.0, \mathrm{P}=0.005$ ). P-value key: ${ }^{\mathrm{P}}$ $<0.05,{ }^{*} \mathrm{P}<0.01,{ }^{* \star *} \mathrm{P}<0.001,{ }^{* \star * \star \mathrm{P}}<0.0001$. 


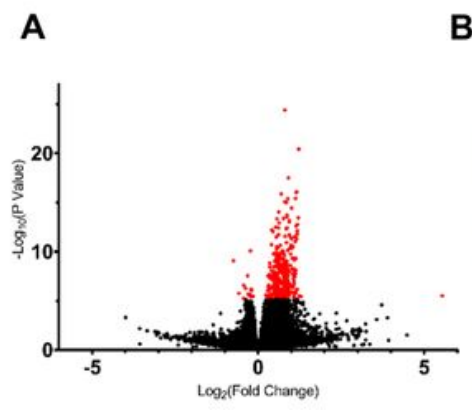

B

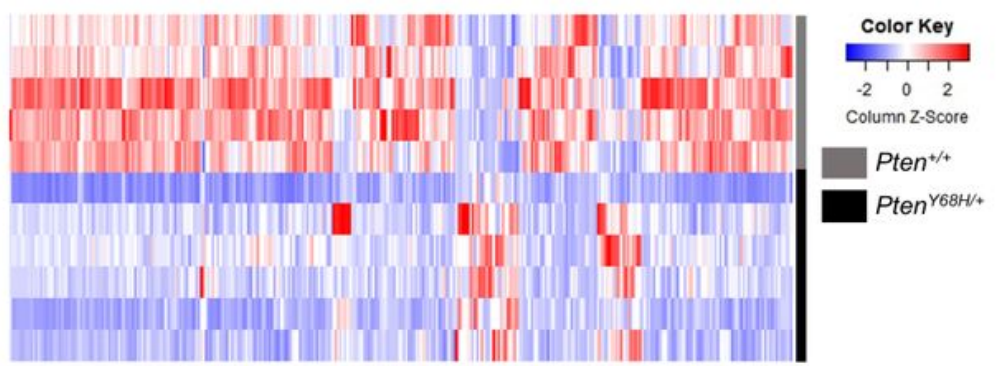

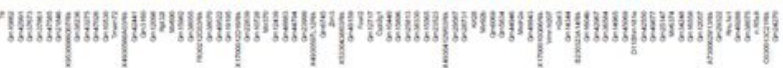

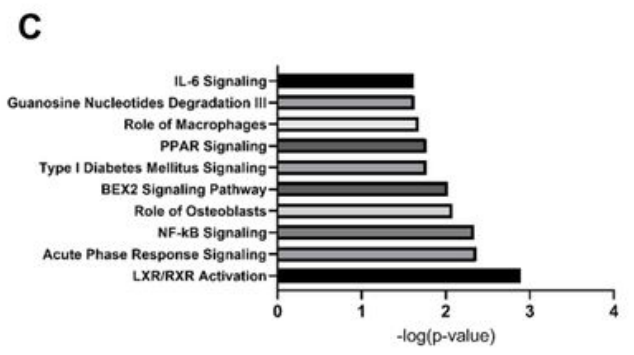

D
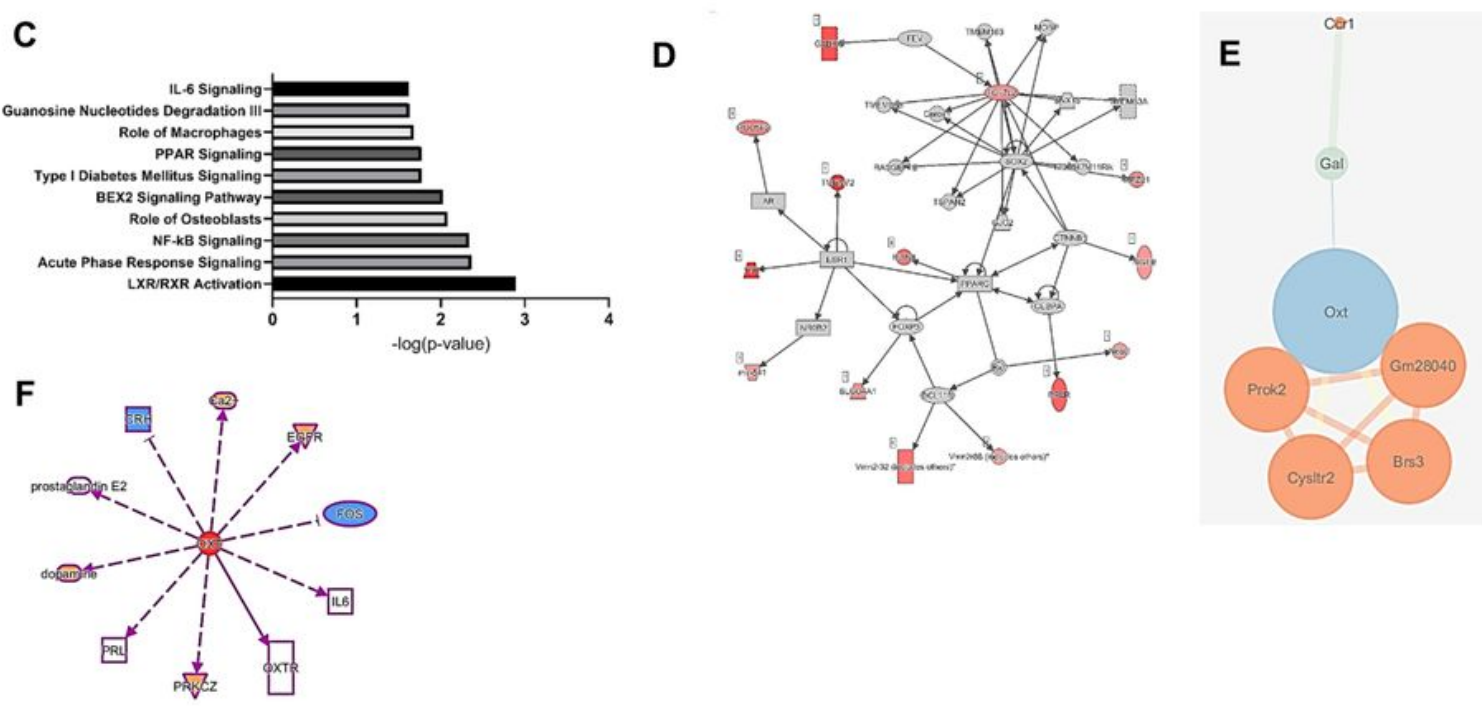

\section{Figure 3}

Transcriptomic survey of Pten $\mathrm{Y} 68 \mathrm{H} /+$ cortex identifies broad changes in expression, affecting PTEN signaling and neurological processes, including oxytocin overexpression. a Volcano plot highlighting genes showing highly significant changes in differential expression $(D E)$ in red $(P<0.0001 ; N W T=6 ; N Y 68 H=5)$. b Heatmap of DE genes (threshold: $P<0.001$ ) clustered by column (i.e., by expression pattern within a gene). c Top "Canonical Pathways" identified by Ingenuity Pathway Analysis (IPA) from DE gene list input. d Top identified IPA network from input DE gene list. The network is organized hierarchically. Red = 
overexpression; green = underexpression; solid line = direct relationship; hashed line $=$ indirect relationship; arrow direction = direction of relationship. e STRING analysis of subnetwork of DE genes displaying the relationship between overexpressed Oxt and other DE genes (i.e., interactome). Node size trends with degree connectivity. Node color trends with betweenness centrality (i.e., cooler colors indicated higher betweenness centrality). Thickness of edge trends with the confidence in the biological relationship. $f$ An oxytocin regulatory network constructed using IPA's Grow and Molecule Activity Predictor (MAP) tools. Blue = predicted inactivation; orange $=$ predicted activation; solid line $=$ direct relationship; hashed line $=$ indirect relationship; arrow direction $=$ direction of relationship.
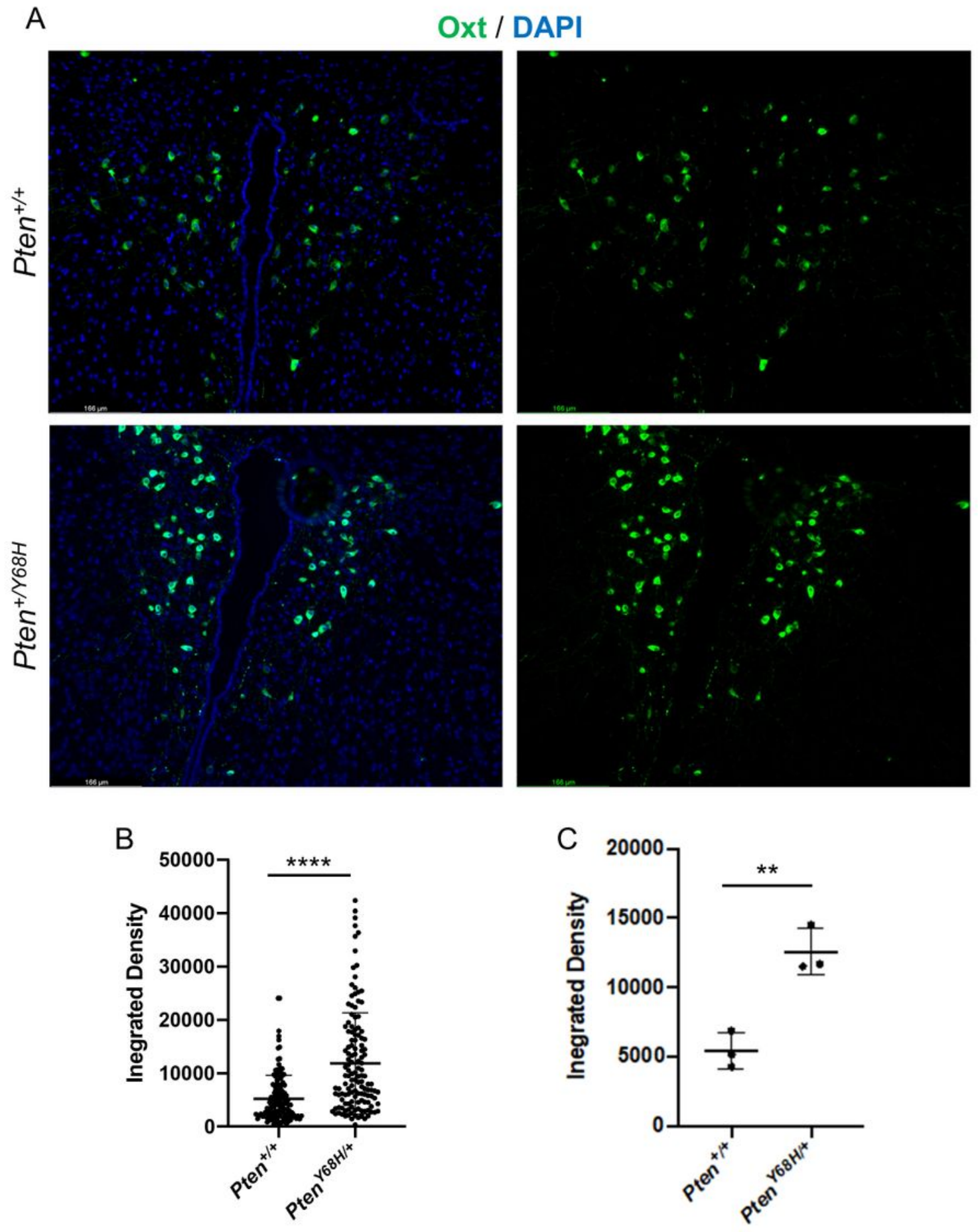


\section{Figure 4}

Overexpression of oxytocin in paraventricular nucleus (PVN) of the hypothalamus of six-week-old Pten $+/+$ and Pten $\mathrm{Y} 68 \mathrm{H} /+$ mice. a Representative immunofluorescence staining of six-week-old Pten+/+ and PtenWT/Y68H PVN for Oxt (green) and DAPI (blue). $\mathrm{N}=3$. Magnification $=10 \mathrm{X}$. Scale bar $=166 \mu \mathrm{m} . \mathrm{b}$ Quantification of the integrated density of Oxt stain per individual PVN cell soma from the image data represented in panel a, finding a significant increase in Oxt expression (Mean $\Delta$ IntDensity $=6630 ; 95 \% \mathrm{Cl}$ : $4860-8400 ; P<0.0001)$. c Quantification of the integrated density of Oxt stain per biological replicate, finding a significant increase in Oxt in the Pten $\mathrm{Y} 68 \mathrm{H} /+\mathrm{PVN}$ compared to Pten+/+ PVN (Median $\triangle$ IntDensity $=7140 ; 97 \%$ Cl: $3700-10600 ; \mathrm{P}=0.0045)$. P-value key: $* \star \mathrm{P}<0.01$ and $\star \star \star \star \mathrm{P}<0.0001$.

\section{Supplementary Files}

This is a list of supplementary files associated with this preprint. Click to download.

- AdditionalFile1Supplementarylnformation.docx

- AdditionalFile2.xlsx 